\title{
A Study of the p-Adic Frobenius Lifts and p-Adic Periods, from a Deformation Theory Viewpoint
}

\author{
Lucian M. Ionescu \\ Mathematics Department, Illinois State University, Normal, IL, USA \\ Email: LMIones@ilstu.edu
}

How to cite this paper: Ionescu, L.M (2018) A Study of the p-Adic Frobenius Lifts and p-Adic Periods, from a Deformation Theory Viewpoint. Advances in Pure Mathematics, 8, 408-418. https://doi.org/10.4236/apm.2018.84023

Received: January 12, 2018

Accepted: April 16, 2018

Published: April 19, 2018

Copyright $\odot 2018$ by author and Scientific Research Publishing Inc. This work is licensed under the Creative Commons Attribution International License (CC BY 4.0).

http://creativecommons.org/licenses/by/4.0/

\begin{abstract}
A canonical p-adic Frobenius lift is defined in the context of p-adic numbers, viewed as deformations of the corresponding finite field. Applications to p-adic periods are considered, including to the classical Euler gamma and beta functions and their $\mathrm{p}$-adic analogues, from a cohomological point of view. Connections between various methods for computing scattering amplitudes are related to the moduli space problem and period domains.
\end{abstract}

\section{Keywords}

p-Adic Numbers, Frobenius Lift, Periods, Feynman Integrals, Deformation Theory

\section{Introduction}

Algebraic integrals called periods [1] [2] [3] ${ }^{1}$, are a new class of numbers extending algebraic numbers and bridging quantum physics and mathematics together, in a way yet to be better understood [5] [6] [7].

Presenting p-adic numbers as deformations of finite fields allows a better understanding of Frobenius lifts and their connection with p-derivations in the sense of Buium [8]. In this way "numbers are functions", as recognized before [9], allowing to view initial structure deformation problems as arithmetic differential equations as in [10], and providing a cohomological interpretation to Buium calculus via Hochschild cohomology which controls deformations of algebraic structures.

A p-adic version for periods has been defined, in quite abstract terms, in connection with Tate-Hodge Conjecture and later on, Fontaine Conjectures [11], but a more "down-to-earth" lucrative approach is yet to be found, in order to

${ }^{1}$ See [4] for additional aspects regarding periods. 
support the "movement" of physics towards an "Ultimate Physics Theory" in terms of Number Theory, as for example the developments of a p-adic String Theory [12] [13], and one which "sounds" more familiar to the physicist, who expects the keyword "quantization" somewhere down the path (and "deformation" can be snicked quite nicely before that!).

From a computer science perspective, instead, a "Quantum Theory" could be formulated already "quantum", i.e. discrete and of finite type, using the language of Quantum Computing at foundations, as a general flow on networks calculus (e.g. Turaev's on Ribbon Categories), at a conceptual level ${ }^{2}$. What still is needed is to understand the role of primes (and Riemann spectrum), in the context of what the present author calls p-adic (finite) strings. But then, a deformation quantization of the theory of finite fields should be beneficial for a brainstorming translation of the "good concepts" from "continuous mathematical-physics over complex numbers, to the discrete graded case of p-adics and adeles". Indeed, if the complex numbers are thought of as the algebraic closure of the topological closure of the rationals $C=\bar{Q}_{\infty}$, then algebraic extensions of $\mathrm{p}$-adic numbers $Q_{q}, q=p^{n}, n \in N$, are enough, even though they are not algebraically closed (the closure $C_{p}$ is much bigger: see [14]).

In this note we will explain how p-adic numbers can be understood as deformations of finite fields as Galois-Klein geometries, in terms close to the spirit of deformation quantization, a perspective hopefully beneficial for incorporating Algebraic Geometry into a Number Theory approach to Finite String Theory.

The article is organized as follows. The p-adic numbers are first reformulated as formal series, in the sense of Deformation Theory, together with basic facts, including how a canonical lift of Frobenius, as a companion deformation of their symmetries [15].

Then the p-derivations forming the basis of the lambda calculus of Buium are reviewed, and reinterpreted, from the deformation point of view. The appearance of Hochschild cohomology is no accident, and allows to address the periods and the period comparison isomorphism in a new light, briefly.

As applications, the article comments on the p-adic analogues of Euler's gamma and beta integrals, which are natural extensions of the Gauss and Jacobi sums in finite characteristic. The connections with scatering amplitudes, as Feynman Integrals and Veneziano amplitudes, well know to be "coincidentaly" related to number theoretic values like Multiple Zeta Values, no longer seem so unexpected, in view of the correspondence between the theories of period isomorphisms.

In conclusion, the author ponders on the similarity between the familiar mathematics of real numbers and that of p-adics "analysis", or rather deformation theory, which substantiates the claim that $\mathrm{p}$-adic numbers are in fact functions, will all their benefits. Finally, further directions of development are discussed, and questions are raised, especially regarding the moduli problem from a De-

${ }^{2}$ Indeed, what are quantization functors on cobordism categories, or spin networks etc. if not that!? 
formation Theory standpoint.

\section{2. p-Adic Numbers as Functions}

The traditional Cauchy completion approach to p-adic numbers $Q_{p}$ hides their alternative algebraic presentation as a $Z$-module of formal series with coefficients in $\left(Z / p Z,+_{p}\right)$, as noted long time ago by Hensel, who compared them with Laurent series:

$$
Z_{p}=\left(F_{p}[[h]], *\right) \ni a=\sum_{i=1}^{\infty} a_{n} h^{n}, a_{n} \in F_{p}, a_{n} * b_{n}=\left(a_{n}+{ }_{p} b_{n}\right)+c_{p}\left(a_{n}, b_{n}\right) h .
$$

We will focus on the "integral elements", since the filed extensions occur as corresponding fraction fields ${ }^{3}$.

Note also that the field structure $\left(F_{p},+, \cdot\right)$ on $\left(Z / p Z,+_{p}\right)$ comes for free as "repeated addition", and that it corresponds to the group of automorphisms as an abelian group (“discrete space of arrows-vectors").

\section{1. p-Adic Deformations of Finite Fields}

The deformation parameter is the generic "Planck's constant" $h$ to avoid the miss-conception that they are "just" the integers completed in the "wrong direction", and to emphasize the conceptual role of the "uniformizer" as a grading parameter. On the contrary, the real numbers result in the topological completion in the wrong direction, of "infinity-small", not needed in a quantum world of atoms and Zeno paradoxes [16]. Note also that the p-adic completion in the direction of the carry-over 2-cocyle

$$
\begin{aligned}
c_{p}(x, y): F_{p} \times F_{p} & \rightarrow F_{p, p}(x, y)=\bmod _{p}(j(x)+j(y))-\left(x+{ }_{p} y\right), \\
& \bmod _{p}: Z \rightarrow F_{p}, j: F_{p} \rightarrow Z,
\end{aligned}
$$

where $\bmod _{p}$ is the reduction modulo $p$ and $j$ is a set theoretical lift of the p-adic digits, has an awkward "closed form" of the deformed addition in terms of a section $j$, as one already knows from the need of introducing Witt vectors [17].

To be more specific, we "zoom in" on the infinitesimal deformation for now, i.e. let us consider the infinitesimal deformation of $Z / p Z$, or equivalently its central extension with 2-cocycle $c_{p}[18]$ :

$$
0 \rightarrow Z / p \rightarrow Z / p^{2} \rightarrow Z / p \rightarrow 0 .
$$

Then the addition modulo $p$ is deformed into a "*-product" as above, to use the physics jargon when comes to quantization via deformation. Indeed, at "tangent space" level (degree 0), the central extension is defined as follows:

$$
\left(a_{0}, 0\right) *\left(b_{0}, 0\right)=\left(a_{0}+b_{0}, c_{p}\left(a_{0}, b_{0}\right)\right),
$$

where we have dropped the subscript from $+_{p}$.

${ }^{3}$ There is a "complication" due to a different convenient definition of integral closure, which is of no concern here. 


\subsection{The Canonical Frobenius Lift}

Having deformed the abelian group $(Z / p Z,+)$, apply a general principle, that an object is determined by its symmetries. Indeed, the Frobenius automorophisms generates the Galois extension $\left[F_{p^{n}}: F_{p}\right]$, so, the deformed structure $Z_{q}=F_{p^{n}}[[h]]$ (with $q=p^{n}$ ), will have automorphisms generated by the corresponding deformation [15].

In order to have a nice commutation relation, we need to use the Teichmüller character in order to replace the natural p-adic digits $a_{i} \in F_{p}$ with deformations which behave better with respect to the addition (and multiplication), and are suited as coefficients for the so called Witt vectors [19], p.14:

$$
Z_{q} \cong W\left(F_{q}\right) \ni x=\sum \tau\left(x_{n}\right) p^{n} .
$$

Requiring to obey the same relations $a_{1}^{p}=a_{i}$, but this time modulo $p$, by Hensel Lemma [20] there are unique $\mathrm{p}$-adic numbers/h-adic series $x_{i} \in Z_{p}$, which is a $(p-1)$-root of unity, satisfying the following initial value problem:

$$
x_{i}^{p}=x_{i}\left(\text { in } Z_{p}\right), \quad x=a_{i} \bmod p\left(\text { in } F_{p}\right) .
$$

Then $\tau\left(a_{i}\right)=x_{i}, a_{i} \in F_{p}$ defines a multiplicative character on $F_{p}^{\times}$, called the Teichmüller character.

Then the canonical lift of Frobenius $\phi$ "commutes" with the Teichmüller character:

$$
\phi_{p}(\tau(v))=\tau(v)^{n}, v \in F_{q}, \quad \phi(x)=\sum \tau\left(x_{n}\right)^{n} p^{n}, x=\sum \tau\left(x_{n}\right) p^{n},
$$

i.e. the Teichmüller character pull-back of the lift of Frobenius is the Frobenius pull-back of the lift of the Teichmüller character.

\section{Relations with Buium Calculus}

As mentioned above, $\mathrm{p}$-adic numbers are analogues of Laurent series, as noticed initially by Hensel, and more recently by Manin [9], idea developed recently into a full-blown calculus by Buium [8].

\section{1. p-Derivations and Lambda Calculus}

Following [10] (specialixed to p-adics framework, with $f(x)=x$ be identity) ${ }^{4}$, a p-derivation $\delta(x)$ on p-adic numbers, thought of as functions conform [9], is a mapping satisfying the following conditions [10], p.2:

$$
\begin{gathered}
\delta(x+y)=\delta_{p}(x)+\delta_{p}(y)+C_{p}(x, y), \quad C_{p}(x, y)=\left[x^{p}+y^{p}-(x+y)^{p}\right] / p, \\
\delta(x y)=x^{p} \delta(y)+\delta_{p}(x) y^{p}+p \cdot \delta_{p}(x) \delta_{p}(y) .
\end{gathered}
$$

It is immediate that any such p-derivation allows to define a ring endomorphism $\phi_{p}(x)=x^{p}+p \delta_{p}(x)$, which is a lift of Frobenius $\phi_{p}(x)=x^{p} \bmod p$ (loc. cit.). This can be understood using Hochschild cohomology, as follows.

${ }^{4}$ Extensive details can be found in [8]. 


\subsection{Relation with Deformation Theory}

With our notation, $p \mapsto h$ and $+\mapsto *$, the cohomological interpretation of the $1^{\text {st }}$ equation above is $d_{\text {Hoch }}^{*} \delta_{p}=-C_{p}$, where the associative operation in view is p-adic addition *, i.e. the deformation of component-wise addition of formal power series in $h$. Of course, $C_{p}$ is itself essentially an exact 2-cocycle (shifted in degree zero):

$$
d_{\text {Hoch }} \operatorname{Frob}(x, y)=\operatorname{Frob}(x)-F(x+y)-\operatorname{Frob}(y)=C_{p}(x, y) p,
$$

so that the Frobenius shift is itself a Hochschild 2-cocycle:

$$
d_{\text {Hосh }} \phi_{p}=d_{\text {Hосh }} F r o b+p d_{\text {Hосh }} \delta_{p}=0,
$$

and the shift is an endomorphism.

The second equation above is, similarly a Hochschild 2-coclye $d_{\text {Hoch }} \delta_{p}=0$, but this time with respect to the multiplicative associative operation, when viewing $Z_{q}$ as an $Z_{q}$-module via the "natural" Frobenius action $L_{x}(y)=x^{n} y$. Now rewrite a p-derivation using our notation, as a Kahler differential 1-form $\omega(x)=\delta(x) d h$, in the formal series deformation parameter $h$ :

$$
\omega=\left[\phi_{p}(x)-\operatorname{Frob}(x)\right] d h / h .
$$

Then it is a Cauchy kernel, i.e. the product of an "entire function" times the generator $d h / h$ of the 1-st (Monski-Washnitzer) algebraic de Rham homology group $H_{M W}^{1}(X)$ (see [21], Ex. 3.1.9, p.28).

The proper reinterpretation of the above formulas in terms of the Teichmüller character and Frobenius lift will be discussed separately [22].

\section{Applications to Periods}

Nevertheless the deformation theory approach sketched above allows for a better understanding of the p-adic periods, in view of a comparison of the MW-cohomology with Hochschild cohomology [23], and corresponding period isomorphism [21] [24].

\section{1. p-Adic Euler Functions Analogues}

The p-adic analogues of Euler's Gamma function, called the Morita Gamma function $\Gamma_{p}$, and the $\mathrm{p}$-adic analog of the Beta function $B_{p}$, are [25]:

$$
\Gamma_{p}=(-1)^{x} \prod_{1<i<x, p \nmid i} i, \quad B_{p}(x)=\Gamma_{p}(a) \Gamma_{p}(b) / \Gamma(a+b)
$$

having the following Hochschild cohomological interpretation Beta $=d_{H} \Gamma$, as in the classical case, over the real numbers.

If an analogue of the p-adic Gauss sum is defined (Equation (4.1) loc. cit. p.362, with $f=1$ for simplicity) $g_{p}(x)=\tau(\theta(x))$, as a composition of Teichmüller character $\tau$ and the exponential $\theta(x)=\exp \left[\xi\left(x-x^{q}\right)\right]$, where $\xi^{p-1}=-1$, then a similar cohomological interpretation of a 2-cocycle holds: $B_{p}=d \Gamma_{p} \quad(\mathrm{p} .366)$.

Before commenting on the role of periods of some special values of these 
functions, we further note that a p-adic analog of the Jacobi sum, naturally deformed via the Teichmüller character (compare with Equation (7.2) [25], p.365, except for the chosen negative sign):

$$
J\left(c, c^{\prime}\right)=\left(c * c^{\prime}\right)(1), \quad c=\tau^{\alpha}, c^{\prime}=\tau^{\beta},
$$

is again, in a consistent way, an exact 2-coboundary $J_{p}=d_{\text {Hoch }} g_{p}{ }^{5}$.

The author's interpretation in the context of Monsky-Weishnizter cohomology, an algebraic de Rham adaptation to the p-adic case, may be understood in the context of period isomorphisms, next.

\subsection{Relation to Period Isomorphisms}

The de Rham period isomorphism for algebraic varieties over complex numbers have various analogues to p-adics, as developed by Tate, Faltings and more recently Fontaine [11], who introduced the so call big $B$-rings of periods [26]. The deformation theory viewpoint adopted above suggests a possible comparison isomorphim can be formulated in terms of Hochschild cohomology, instead of, and corresponding to the etale cohomology comparison isomorphism as usual. A starting point, only, would be a cohomological interpretation of Rohrlich formula for the periods of Fermat curves $F_{m}: x^{m}+y^{m}=1$ in the complex case, as a product of a Hochschild 2-coboundary and a cyclotomic number [27], \$2, p.2:

$$
\begin{gathered}
\int_{\gamma} \eta_{(r / m, s / m)} \in B(r / m, s / m) \cdot Q\left(\zeta_{m}\right), \\
\gamma \in H_{1}\left(F_{m}(C), Q\right), \quad \eta_{(r / m, s / m)}=d x^{r} / y^{m-s} \in H_{d R}\left(F_{m}(C), Q\right),
\end{gathered}
$$

where $s, t \in Z / m Z$, and $s+t=1$ to suggest the underlying convolution, as in a Jocobi sum or Euler beta function, with cohomological significance.

The p-adic analog for formula for the p-adic periods allows to infer that the image of the p-adic comparison cohomology pairing has a smaller image that the "big" Fontaine B-rings (loc. cit. p.3):

$$
H_{1}\left(F_{m}(C), Q\right) \times H_{d R}^{1}\left(F_{m}, Q\right) \rightarrow B_{d R} .
$$

It is interesting to ponder on a possible interpretation of the p-adic periods derived from the action of the Frobenius lift on cohomology, conform to Lefschetz Fixed Point Theorem [21], p.28:

$$
\# \bar{X}\left(F_{q}\right)=q^{n-1} \sum_{0}^{n-1}(-1)^{\mathrm{T}} r\left(\left(\phi^{*}\right)^{-1}\left|H^{i}\right| M W(\bar{A})\right),
$$

yielding a possible connection between its eigenvalues, the "Weil zeros" satisfying the p-adic analog of Riemann Hypothesis, and p-adic periods.

\subsection{Relation to Veneziano Amplitude}

The formal analogy with the Jacobi sum may be further strengthen by considering the above p-adic Jacoby sum $J_{p}=d_{\text {Hoch }} g_{p}$, a 2-characters convolution val- 
ue.

The Veneziano's “educated guess” for a Regge trajectory compatible scattering amplitude [28] [29], which in some sense "started" the String Theory movement, is an iterated integral on moduli spaces of punctured Riemann spheres $\mathcal{M}_{0,4}$ [30], Equation (1.1), p.1, with $n=1$ :

$$
A(4 \text {-points })=B(a, b)=\left(d_{H o c h} \Gamma\right)(a, b)=\int_{0 \leq t \leq 1} t^{a}(1-t)^{b} \mathrm{~d} t,
$$

and hence a period. This can be related to Multiple Zeta Values, explaining in an indirect way the "coincidence" with the Feynman amplitudes, as linear combinations of MZVs [31], "closing”, in a way, this circle of ideas.

It suggests that perhaps an analogous moduli space for $\mathrm{p}$-adic curves exists (beyond the scope of this article), or at least a direct connection with the Multiple Zeta Values [32], and opening a research direction for explaining the "unreasonable" effectiveness of the new methods for computing scattering amplitudes, starting with the BCFW-recursion method [33], towards the general, yet elusive as for now, amplituhedron concept [5] [22] [34].

\section{Conclusions and Further Developments}

As an overarching theme, quantization was always thought of as a sort of "deformation" of Newtonian physics in the "direction" of Planck's constant, not to mention the other deformation, of Galilean Relativity in the direction of $1 / \mathrm{c}$, the inverse of the speed of light.

That Deformation Theory is a natural generalization of Lie Theory from the framework of Lie algebras/Lie groups to quite general algebraic structure [35], including the modern mathematics of Quantum Groups, and should be enough incentive for "prefering" to advertise p-adic "analysis" as p-adic deformation theory.

The interplay between algebraic and analytic, pertaining to field extensions $F_{q}$ and h-adic completions $Z_{q}$, with their fields of fractions $Q_{q}^{6}$, can be pictured as a 2 -dimensional $1^{\text {st }}$ octant grid of deformations of "vector bundles":

(Algebraic)

Extensions
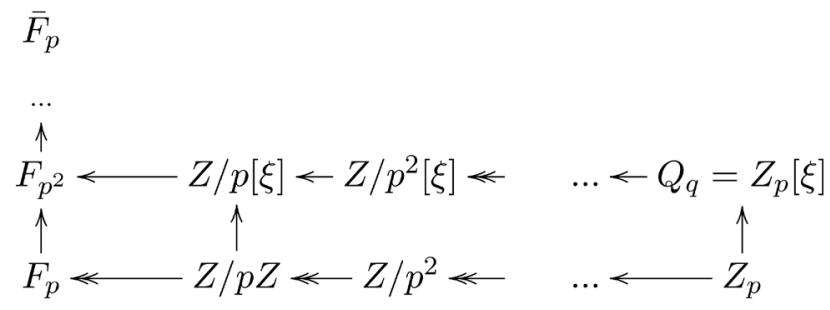

(Algebraic) Deformations

Then the similarity between "real/complex (topologically completing the rationals at the place/prime $p=\infty$ ) vs. $\mathrm{p}$-adic mathematics is obvious:

${ }^{6} \ldots$ perhaps better projectively completed as $Z_{q} P^{\mathrm{l}}$ for Algebraic Geometry treatments. 


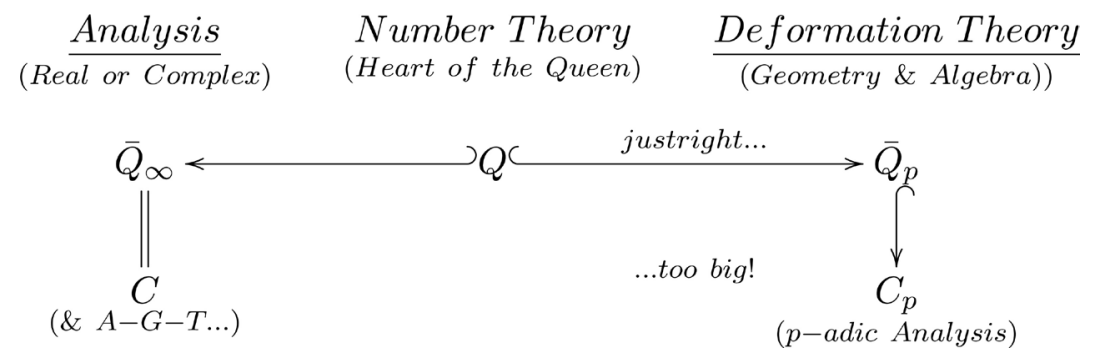

and the "Hamletian" question "To use graded or non-graded structures?" has an obvious answer, since graded structures often come with benefits, e.g. the antipode of a graded bialgebra.

The advantage of the p-adics side, of being graded mathematics, is that numbers can be treated as functions. the historical connection with the integers may be broken, and $p$-adic "numbers" are just $h$-adic Laurent series, so that the corresponding fields $Q_{q}$ may be treated both analytically and as number fields. The fact that they are not algebraically complete is not an issue, since extensions should be rather viewed as objects of a category, rather than as a big huge one-object of study.

On the concrete side, the deformation theory point of view allows to proficiently make use of Hochschild cohomology, as the one that controls deformations. The various p-adic analogues of special functions, starting with the Gamma Function $\Gamma$, which in fact perhaps should be thought of as the Melin transform of the exponential, in the context of Fourier Duality ${ }^{7}$, and the derived 2-cocycle, the Euler Beta function, have p-adic analogues, which seem to have deeper significance in this context.

The periods from Quantum Physics, via Feynman Integrals, MZV, have p-adic analogues if we take Veneziano Amplitude as a model, and recall that it is an iterated integral on an algebraic variety with a divisor (Riemann sphere with marked points). Therefore the "translation" of the theory of periods in p-adic realm is perhaps easier, avoiding the big $B$-period rings of Fontaine, of course, by non-experts.

Returning to the underlying theme, that "Deformation Theory is the study of infinitesimal conditions associated to varying a solution $P$ of a problem ...” [36], explains in a way why the perturbative approach via Feynman diagrams and integrals should yield periods, and that moduli spaces are, again in some sense, period domains parameterizing deformations controlled by cohomologies. This justifies why periods coming in families usually satisfy differential equations even in the arithmetic setup, as for example the one studied in [10]

Also, since deformations are classified by some cohomology groups, and in particular algebraic deformations like the p-adic numbers and their endomorphisms, are characterized by Hochschild 2-cocycles with two deformations isomorphic if cohomologous [23], p.10, Frobenius lifts as deformations of the cor-

${ }^{7}$ The "paradigm" here is pairing additive and multiplicative characters, i.e. harmonic analysis of symmetries. 
responding symmetries are controlled by 1-cohomology classes determined by derivations, like the p-derivations of Buium calculus.

On a final note, understanding the adeles, i.e. how the p-adics deformations glue altogether, on the other hand, is a different story, yet Deformation Theory related, since it is perhaps about the duality of the algebraic quantum group of rationals [37].

\section{Acknowledgements}

I would like to express my gratitude for the excellent research conditions at I'Institut des Hautes Etudes Scientifiques, and for the help from Illinois State University Department of Mathematics, especially Prof. Gail Yamskulna, which made this trip possible.

The discussions with Prof. Kontsevich had a most needed guiding role, clarifying many questions of the present author, and providing extensive additional material, which only partially was used in this article; the "straying away" from the shown path is entirely due to author's childish attempts to explore on his own this new marvelous territory were Mathematics and Physics become one.

The encouragements from Prof. Yuri Manin, and the references provided (and some yet to be followed), confirming that, yes, "numbers are functions", played an important role in pursuing this line of thought.

Finally, the author also needs to thank some old friends, particularly Prof. Yan Soibelman for the lessons on Deformation Theory, and of course, Professors Louis Crane and David Yetter for TQFTs and all that jazz, knotted and braided.

\section{References}

[1] Kontsevich, M. (2006) Periods. http://www.ihes.fr/ maxim/TEXTS/Periods-short.pdf

[2] Muller-Stach, S. (2014) What Is a Period. AMS, 61, 898-899. https://doi.org/10.1090/noti1159

[3] Carlson, J. and Griffiths, P. (2008) What Is a Period Domain? AMS, 55, 1418.

[4] Kontsevich, M. and Zagier, D. (2001) Periods. http://www.ihes.fr/ maxim/TEXTS/Periods.ps

[5] Brown, F. (2015) Periods and Feynman Amplitudes. https://arxiv.org/pdf/1512.09265.pdf

[6] Schnetz, O. (2008) Quantum Periods: A Census of $\phi^{4}$-Transcendentals. Communications in Number Theory and Physics, 4, 1-48. https://doi.org/10.4310/CNTP.2010.v4.n1.a1

[7] Ionescu, L.M. and Sumitro, R. (2017) Periods and Applications. arXiv:1708.09277 [math.HO]

[8] Buium, A. (2005) Arithmetic Differential Equations. Mathematical Surveys and Monographs Volume 118, American Mathematical Society, Providence, 310 p. https://doi.org/10.1090/surv/118

[9] Manin, Y.I. (2013) Numbers as Functions. p-Adic Numbers, Ultrametric Analysis, and Applications, 5, 313-325. https://doi.org/10.1134/S2070046613040055

[10] Buium, A. and Manin, Y.I. (2013) Arithmetic Differential Equations of Panleve VI 
Type. arXiv:1307.3841 [math.NT]

[11] Wikipedia (2017) p-Adic Hodge Theory. https://en.wikipedia.org/wiki/P-adic_Hodge_theory

[12] Volovich, A. (2010) Number Theory as the Ultimate Physics Theory. p-Adic Numbers, Ultrametric Analysis, and Applications, 2, 7787. https://doi.org/10.1134/S2070046610010061

[13] Dragovich, B., Khrennikov, A.Yu., Kozyrev, S.V., Volovich, I.V. and Zelenov, E.I. (2017) p-Adic Mathematical Physics: The First 30 Years. p-Adic Numbers, Ultrametric Analysis, and Applications, 9, No. 2, 87-121. https://doi.org/10.1134/S2070046617020017

[14] Koblitz, N. (1984) p-Adic Numbers, p-Adic Analysis, and Zeta-Functions. Graduate Texts in Mathematics, Springer, New York.

[15] Wikipedia (2018) Galois Deformations. https://en.wikipedia.org/wiki/Deformation_theory\#Galois_deformations

[16] Wildberger, N.J. (1999) Real Fish, Real Numbers, Real Jobs. The Mathematical Intelligencer, 21, 4-7.

[17] Finkel, D. (2007) An Overview of Witt Vectors. https://wstein.org/wiki/attachments/ant07(2f)projects/finkel-witt_vectors.pdf

[18] Gerstenhaber, M. (1964) On the Deformation of Rings and Algebras. Annals of Mathematics, 79, 59-103. https://doi.org/10.2307/1970484

[19] Rabinoff, J. (2014) The Theory of Witt Vectors. https://arxiv.org/pdf/1409.7445.pdf

[20] Wikipedia, Hensel's Lemma. https://en.wikipedia.org/wiki/Hensel\%27s_lemma

[21] Hartog, R. (2017) Zeta Functions and Dwork Modules. MS-Thesis, Utrecht University, Utrecht.

[22] Ionescu, L.M. (2018) Periods, Feynman Integrals and Jacobi Sums. IHES (Work in Progress).

[23] Anel, M. (2013) Why Deformations Are Cohomological. http://mathieu.anel.free.fr/mat/doc/Anel\%20-\%20WhyDeformationAreCohomolog ical.pdf

[24] Kedlaya, K.S. (2001) Counting Points on Hyperelliptic Curves Using Monsky-Washnitzer Cohomology.

[25] Boyarsky, M. (1980) p-Adic Gamma Functions and Dwork Cohomology. Transactions of the American Mathematical Society, 257, 359-369.

[26] Jean-Marc, F. (1994) Priodes p-Adiques, Astrisque, 223. Socit Mathmatique de France, Paris.

[27] Kashio, T. (2011) On a Relation between p-Adic Gamma Functions and p-Adic Periods. Workshop on L-Functions. http://www2.math.kyushu-u.ac.jp/ weng/kashio.pdf

[28] Wikipedia, Veneziano Amplitude. https://en.wikipedia.org/wiki/Veneziano_amplitude

[29] Kholodenko, A.L. (2005) New Strings for Old Veneziano Amplitudes: I Analytical Treatment. https://arxiv.org/pdf/hep-th/0410242.pdf

[30] Bogner, C. and Brown, F. (2014) Feynman Integrals and Iterated Integrals on Moduli Spaces of Curves of Genus Zero. https://arxiv.org/pdf/1408.1862.pdf

[31] Hartnett, K. (2016) Strange Numbers found in Particle Collisions. Quanta Magazine. 
https://www.quantamagazine.org/strange-numbers-found-in-particle-collisions-201 $61115 /$

[32] Brown, F. (2006) MZVs and Periods on the Moduli Spaces. https://arxiv.org/abs/math/0606419

[33] Britto, R., Cachazo, F., Feng, B. and Witten, E. (2005) Direct Proof of Tree-Level Recursion Relation in Yang-Mills Theory. Physical Review Letters, 94, Article ID: 181602.

[34] Arkani-Hamed, N. and Trnka, J. (2007) The Amplituhedron.

[35] Ionescu, L.M. (2010) From Lie Theory to Deformation Theory. International Journal of Pure and Applied Mathematics, 58, 209-230.

[36] Wikipedia, Deformation Theory. https://en.wikipedia.org/wiki/Deformation_theory

[37] Van Daele, A. (1998) An Algebraic Framework for Group Duality. Advances in Mathematics, 140, 323-366. https://doi.org/10.1006/aima.1998.1775 